The Impact of Large-Scale Surveys on Pulsating Star Research

ASP Conference Series, Vol. 203, 2000

L. Szabados \& D. W. Kurtz, eds.

\title{
Hydrodynamical Modeling of Double-Mode Pulsation
}

\author{
Zoltán Kolláth \\ Konkoly Observatory, H-1525 Budapest, P.O. Box 67, Hungary
}

\begin{abstract}
The hydrodynamical modeling of steady double-mode (DM) pulsations has been a long-standing quest. Recent improvements in turbulent-convective pulsation codes provide a natural way to calculate nonlinear DM oscillations. The eddy viscosity plays a crucial role in these models. We present models both for RRd and beat Cepheid variables and for different chemical compositions. Simple nonresonant amplitude equations capture the DM phenomenon, giving an overview of modal selection on the HR diagram.
\end{abstract}

\section{Introduction}

Double-mode (DM) pulsation is common among both Cepheids and RR Lyrae stars. The recent large-scale surveys have discovered even more DM variable stars in the Magellanic Clouds. Those pulsators are very important in verifying pulsation theory and the numerical models, since the double periodicity and the view of global modal selection provide many more observational constraints than their single mode siblings. However, the efforts to model DM pulsation has remained unsuccessful until very recently. Although purely radiative models of RRd stars were found already in 1993 by Kovács and Buchler, those results did not give a general solution to the problem due to the lack of a physical dissipation in the models. DM behavior in RR Lyrae (Feuchtinger 1998) and Cepheid models (Kolláth et al. 1998) were found simultaneously, but independently, by the Vienna and the Florida codes. The breakthrough was induced by the inclusion of time-dependent turbulent convection in the models.

\section{Methods}

The pulsation code we used for the calculations is discussed by Buchler (2000; see also Yecko, Kolláth, \& Buchler 1998). Here we mention only that the free parameters in the code are not yet set. For this reason we do not make a detailed comparison between observations and models. However, the tendencies (e.g. along a sequence with different temperatures) can be used as primary tests on our models.

Claiming double-mode behavior is not an easy task. Very long transients with mixed mode behavior exist in the hydro calculations, even when the model converges to a single mode solution (limit cycle). This means that synthetic 


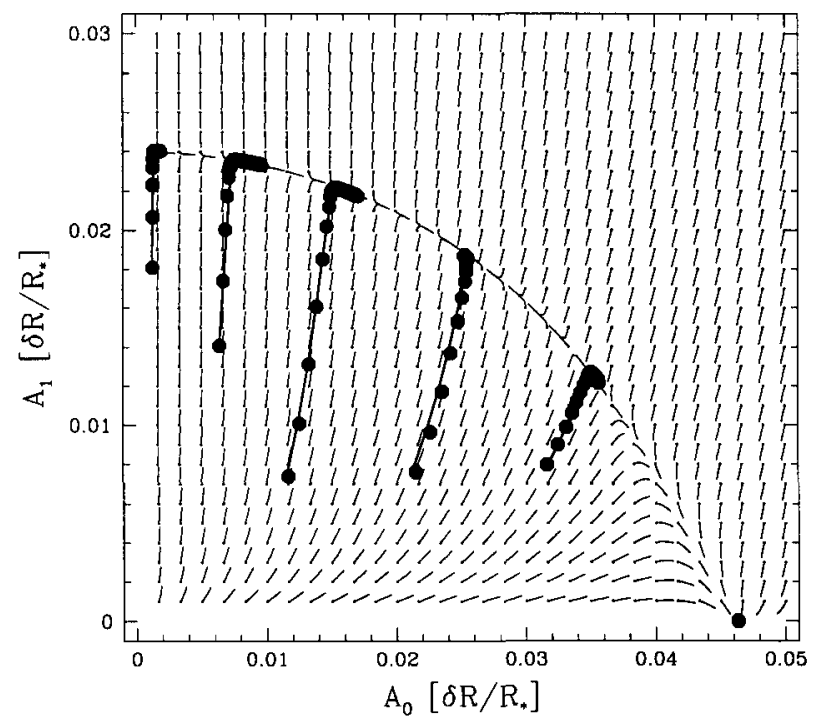

Figure 1. The flow in the $A_{0}-A_{1}$ phase space. The large dots represent the hydro results. The thick lines are the solutions of the amplitude equations giving the best fit to the data.

velocity and light curves or Fourier spectra from single model calculations cannot provide a proof of steady DM behavior.

A great advantage of the Florida code is that it can relax to the periodic solutions by iteration and it provides the stability (Floquet) analysis of the limit cycles. When both the fundamental mode and the first overtone are linearly unstable but the other modes are stable, then Floquet exponents of their limit cycles provide the primary information on modal selection. If both periodic solutions are unstable against perturbations, then the model should oscillate in a DM state only. However, stable double-mode solutions can exist even when one of the single mode cycles is stable, as our results indicate. Then the Floquet analysis is not sufficient to identify DM pulsations.

A more time consuming, but robust method is to run the same model with different initial perturbations of the static model and to extract the slowly varying amplitudes with the help of a time-dependent Fourier decomposition. The resulting phase portraits $\left(A_{1}(t)\right.$ vs. $\left.A_{0}(t)\right)$ then can be used to examine the possible behavior of the given model. In Fig. 1 we show a representative set of hydro runs for a Cepheid model. The initial perturbations were constructed by a mixture of the fundamental and the first overtone eigenvectors. Fig. 1 provides conclusive proof of the presence of stable beat pulsation that coexists with the stable fundamental mode. As we will see in the next section, in this case the actual state of the star depends on its former evolutionary path. Two other nontrivial, but unstable fixed points exist for the given model in the phasespace of the amplitudes, the first overtone limit cycle and a DM saddle point. The observed behavior of the model can be explained on a fundamental level 


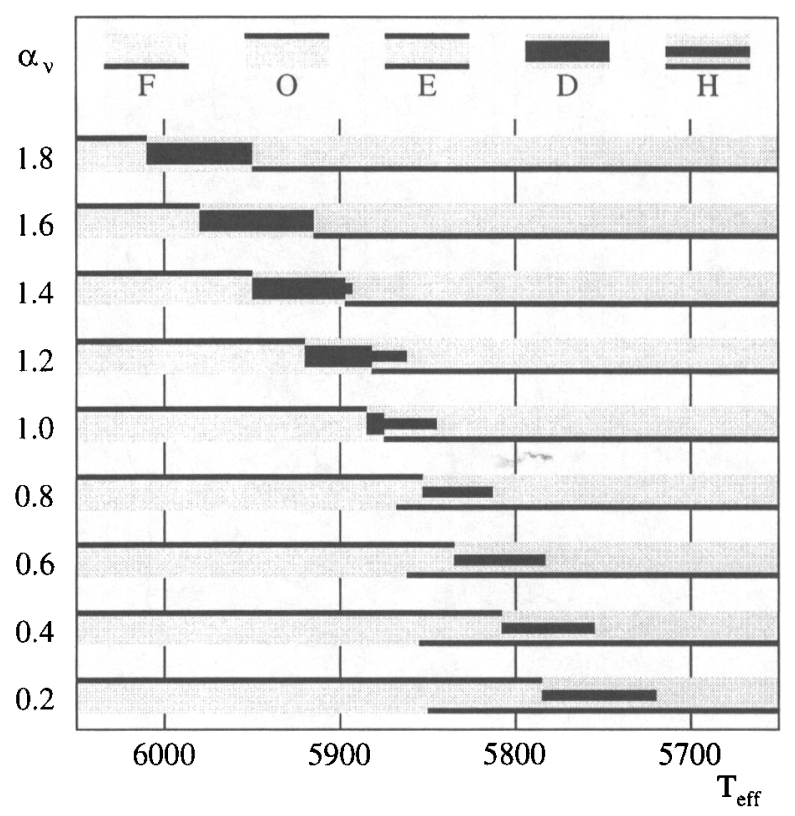

Figure 2. Modal selection on the $T_{\text {eff }}-\alpha_{v}$ plane. The symbols of the modal states are described at the top of the figure.

by amplitude equations (AEs) (Buchler et al. 1999; Kolláth et al. 2000). The thick solid lines in Fig. 1 represent the solutions of the AEs fitted to the set of data. The AEs also can be used to calculate all the fixed points, even if the hydro runs do not reach them. The flow obtained from the amplitude equations is represented by the short thin lines.

The described method together with the Floquet analysis provides the possible states of a given model and the stability of those solutions. Then by repeating the calculations with different effective temperature and luminosity values one can map the whole picture of modal selection on the HR diagram.

\section{Results}

We have run Cepheid models with Galactic and SMC chemical compositions - in both cases F/O1 DM models appears quite naturally. The behavior of the individual models can be classified into five groups: 1) first overtone only $(0)$, 2) fundamental mode only $(\mathrm{F}), 3$ ) either fundamental or first overtone (E), 4) double-mode only (D), and 5) either fundamental or double-mode (H). We have not found any model where the first overtone and the DM fixed points are stable simultaneously, but in principle it can also be possible. For models with ' $\mathrm{E}$ ' or ' $\mathrm{H}$ ' characteristics one should examine a sequence along the possible evolutionary path of the star to predict its pulsational state. Sequences with different temperatures, but with a fixed luminosity, give good estimates for that 


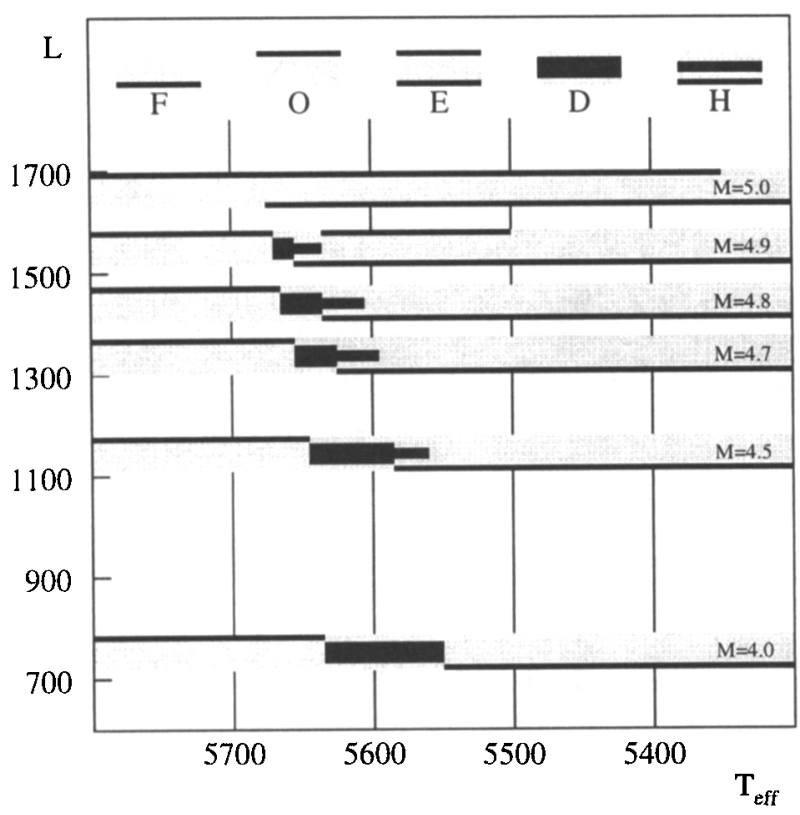

Figure 3. Modal selection of galactic Cepheid sequences on the HRD. The mass of the models is indicated on the right side of the stripes.

purpose. In the sequences with decreasing temperature we could identify the following variations of modal behavior: O-E-F, O-D-F, O-D-H-F, O-E-H-F and even a O-D-H-E-F sequence. For the O-D-F type of sequence DM behavior occurs independently of the direction of evolution. However, for the $\mathrm{O}-\mathrm{E}-\mathrm{H}-\mathrm{F}$ case DM pulsation can be observed only if the star evolves from the blue side of the instability strip to the red. In that case the star evolves to the DM state, when the $\mathrm{O} 1$ mode loses its stability, while in the opposite evolutionary path the star moves to the overtone directly from the $\mathrm{F}$ mode at the blue edge of the fundamental mode instability strip through a transient DM state.

In Fig. 2 the dependence of the modal selection on the eddy viscosity is shown for a Cepheid sequence with SMC composition. We obtained very similar behavior for the Galactic Cepheids and also for the RR Lyrae models. The viscosity has two dominant effects. The first one is that the transition region is shifted to higher temperatures (and to shorter periods) at higher $\alpha_{\nu}$ values. The second one is the changes in the characteristics of modal selection. For high viscosity parameters a DM-only solution exists sandwiched between the $\mathrm{O}$ and $\mathrm{F}$ state (O-D-F). But with decreasing $\alpha_{\nu}$ the characteristics first change to $\mathrm{O}-\mathrm{D}-\mathrm{H}-\mathrm{F}$, then $\mathrm{O}-\mathrm{E}-\mathrm{H}-\mathrm{F}$.

The modal selection on the HRD is demonstrated for Galactic Cepheid models (Fig. 3), and for RR Lyrae models (see Szabó et al. 2000). We calculated the models along a mass-luminosity relation (the masses of the models are indicated at the right end of the gray stripes). A very interesting feature is the disappearance of the DM solution at higher luminosities, even where stable first 

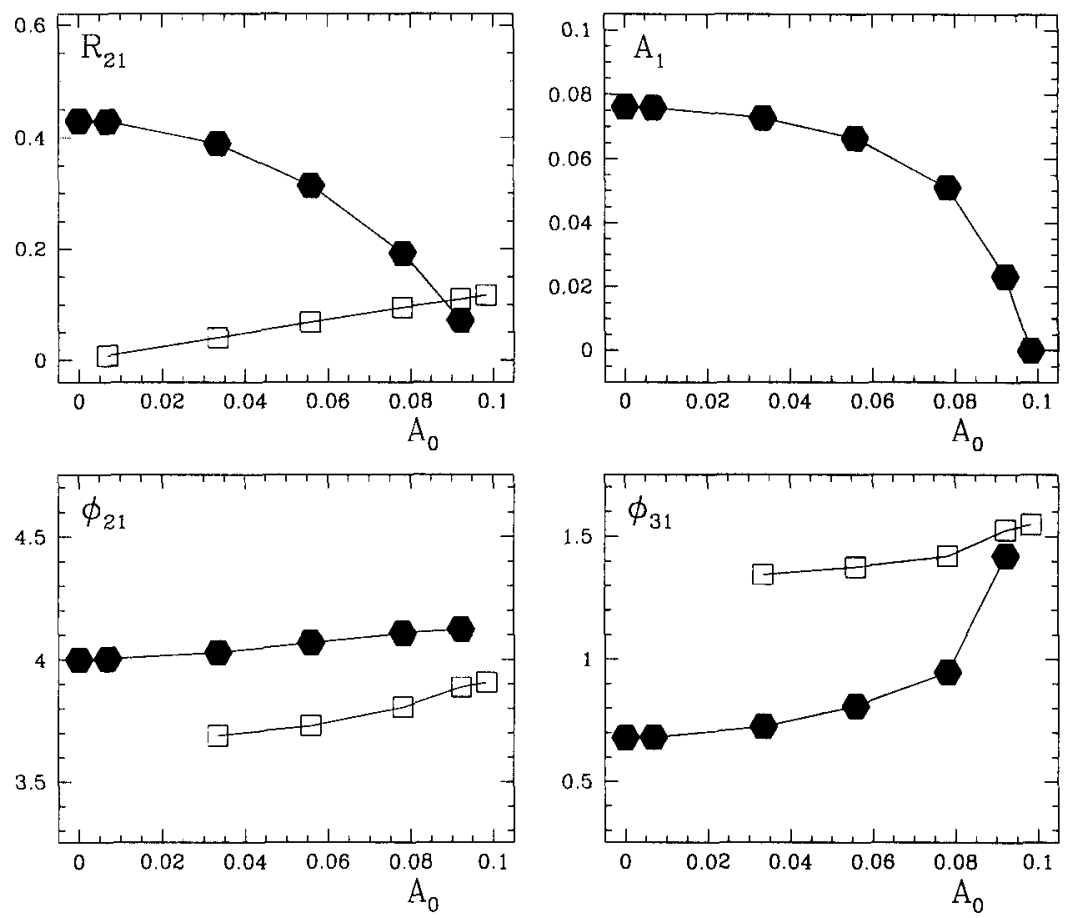

Figure 4. The dependence of the Fourier parameters on the fundamental mode amplitude along the separatrix. Filled hexagons: fundamental mode, open squares: first overtone. The top right box shows the models on the $A_{0}-A_{1}$ phase space.

overtone pulsation exists. This result is in nice agreement with the observations by Udalski et al. (1999).

It was found that the Fourier parameters ( $\phi_{21}$ and $\phi_{31}$ ) of both modes in DM Cepheids are very similar to those of the corresponding modes in monoperiodic stars (e.g. Udalski et al. 1999). Since the models relax very slowly on the separatrix connecting the $\mathrm{F}$ mode to the $\mathrm{O} 1$ through the $\mathrm{DM}$ solution, one can calculate the Fourier parameters along the separatrix. This test provides a clue how the Fourier phases depend on the modal amplitudes (Fig. 4). We selected the model from the middle of the DM region, but we obtained very similar results in the whole region. The Fourier parameters were calculated from the bolometric light curves, and the amplitudes are in mag. To indicate the position on the separatrix, we also show the $A_{1}$ vs. $A_{0}$ plot of the models on the top right box. As expected the $R_{21}$ amplitude ratios have a strong dependence on the corresponding amplitudes. For the fundamental mode this dependence is almost exactly linear. The plots of the $\phi_{21}$ Fourier phases are very flat. The difference between the $\phi_{21}$ value of the DM solution and that of the single mode 
limit cycle is less than 0.2 . The $\phi_{31}$ parameters have the same tendencies but with a little bit stronger amplitude dependence for the $\mathrm{F}$ mode.

The large-scale surveys have found second overtone to first overtone $(\mathrm{O} 2 / \mathrm{O} 1)$ DM pulsators at similar population as $\mathrm{O} 1 / \mathrm{F}$ beat Cepheids. The models should reproduce this constraint too. With the recent parameter setting we could find some $02 / \mathrm{O} 1$ models, but in a very narrow range of temperature and turbulence parameters. To model these DM variables satisfactorily, we have to explore the parameter space in a wider range and probably include further corrections in the turbulent convection recipe. The consistent modeling of DM oscillations in both Cepheids and RR Lyrae stars and with different chemical compositions would be an important step in understanding the physical mechanisms inside the stars.

Acknowledgments. This work has been supported by the Hungarian OTKA (T-026031) grant and by the National Science Foundation (AST9819608). It is a great pleasure to thank J. R. Buchler, Z. Csubry, R. Szabó, and P. A. Yecko for valuable collaboration on this project.

\section{References}

Buchler, J. R. 2000, in these proceedings, p. 343

Buchler, J. R., Yecko, P., Kolláth, Z., \& Goupil, M. J. 1999, in ASP Conf. Ser. Vol. 173, Theory and Tests of Convection in Stellar Structure, ed. A. Gimenez, E. F. Guinan, \& B. Montesinos (San Francisco: ASP), 141 [astro-ph/9901188]

Feuchtinger, M. U. 1998, A\&A, 337, 29

Kolláth, Z., Beaulieu, J. P., Buchler, J. R., \& Yecko, P. 1998, ApJ, 502, L55

Kolláth, Z., Buchler, J. R., Szabó, R., \& Csubry, Z. 2000, A\&A, in preparation

Kovács, G. \& Buchler, J. R. 1993, ApJ, 404, 765

Szabó, R., Kolláth, Z., Csubry, Z., \& Buchler, J. R. 2000, in these proceedings, p. 374

Udalski, A., Soszyński, I., Szymański, M., et al. 1999, Acta Astron., 49, 1 [astro-ph/9903391]

Yecko, P. A., Kolláth, Z., \& Buchler, J. R. 1998, A\&A, 336, 553 